\section{Structural changes of fovea during remission of Behçet's disease as imaged by spectral domain optical coherence tomography}

\begin{abstract}
Purpose To acquire the image of the fovea during remission of ocular Behçet's disease using spectral domain optical coherence tomography (SD-OCT) and to further assess the correlation between best-corrected visual acuity (BCVA) and SD-OCT findings. Methods A total of 24 eyes of 14 patients with ocular Behçet's disease were examined with SD-OCT. The relationships between BCVA and other clinical characteristics, including foveal thickness and status of external limiting membrane (ELM) or the junction between photoreceptor inner and outer segments (IS/OS), were analysed for statistical significance. Results BCVA in eyes with intact IS/OS was significantly better than that in eyes with ill-defined IS/OS $(P=0.0001)$. Foveal thickness $(P=0.0001)$ and male sex $(P=0.03)$ were also significantly different between eyes with and without complete IS/OS line.

Conclusions Definition of the IS/OS line by SD-OCT, but not status of the ELM line, appears to be associated with BCVA in patients with ocular Behçet's disease.

Accordingly, SD-OCT may prove useful in the evaluation of visual function after ocular attacks of Behçet's disease.
\end{abstract}

Eye (2010) 24, 969-975; doi:10.1038/eye.2009.231; published online 25 September 2009

Keywords: uveitis; panuveitis; retinal vasculitis; ocular inflammation; the third high-reflectance band

\section{Introduction}

Behçet's disease is a chronic systemic inflammatory disorder that affects multiple
N Unoki, K Nishijima, M Kita, R Hayashi and $\mathrm{N}$ Yoshimura

organs with generalized vasculitis. ${ }^{1,2}$ Ocular involvement in Behçet's disease is characterized by recurrent episodes of intraocular inflammation or uveitis, most commonly presenting as retinal vasculitis. ${ }^{3}$ In addition, some reports suggest that choroidal abnormalities can be observed by indocyanine green angiography (ICGA) and fluorescein angiography (FA). ${ }^{4-6}$ Although recurrent ocular attacks may lead to irreversible alterations of the sensory retina, which are significant causes of poor visual prognosis in affected patients, ${ }^{7}$ to the best of our knowledge, details of the structural changes in affected retinas have not been documented.

Optical coherence tomography (OCT) is now used widely to detect changes in the retinal architecture of eyes affected by various macular diseases and to measure quantitatively the retinal thickness. With the use of highresolution and contrast, OCT also allows us to evaluate the junctions between inner and outer segments (IS/OS) of the photoreceptors, which have been reported to have a close relationship with visual function in various conditions, including macular hole, ${ }^{8,9}$ branch retinal vein occlusion, ${ }^{10,11}$ and retinal detachment. ${ }^{12}$ Because drastic changes in macula, for example, macular oedema, retinal haemorrhage, or ischaemia are seen in active phase of Behçet's disease, the status of IS/OS line on OCT in Behçet's eye is attracting a great deal of attention as a possible hallmark of the integrity of the outer photoreceptor layer. With this powerful instrument, however, opacity of ocular media prevents us to evaluate the status of the macula in inflammatory phase. The purpose of this study was to describe details of structural changes in the fovea in patients with ocular
Department of Ophthalmology, Kyoto University Graduate School of Medicine, Kyoto, Japan

Correspondence: K Nishijima, Department of Ophthalmology, Kyoto University Graduate School of Medicine, 54 Shogoin kawahara-cho, Sakyo-ku, Kyoto 606-8507, Japan. Tel: + 81757513253 ; Fax: + 81757520933

E-mail: nissi@

kuhp.kyoto-u.ac.jp

Received: 13 February 2009 Accepted in revised form: 11 August 2009 Published online: 25 September 2009 
Behçet's disease that was in remission, using spectral domain OCT (SD-OCT) and to assess their relations to visual acuity.

\section{Methods}

We reviewed the medical records of 24 eyes of 14 patients who had experienced acute ocular inflammation associated with Behçet's disease and were now in remission/inactive phase, and who were examined at Kyoto University Hospital between November 2006 and October 2007. We defined the remission phase as the status without both apparent anterior and posterior inflammation (ie, keratic precipitate, hypopyon, retinitis, vasculitis, vitreous opacity, papillitis, macular oedema and so on). Eyes with other ocular diseases (ie, glaucoma, cataract, or diabetic retinopathy) were excluded. The study described herein was approved by the ethics committee of Kyoto University Graduate School and Faculty of Medicine and adhered to the tenets of the Declaration of Helsinki Principles; informed consent for inclusion in the study was obtained from each patient.

All patients had undergone a complete ophthalmic examination, including best-corrected Snellen visual acuity (BCVA), slit-lamp biomicroscopy, funduscopy, applanation tonometry, and SD-OCT. BCVA values were converted into the logarithm of the minimal angle of resolution (logMAR) for statistical analysis.

We used an SD-OCT (3DOCT-1000; Topcon, Tokyo, Japan) to assess retinal microstructure and to measure retinal thickness. SD-OCT scans were performed with 128 horizontal B scans (each of which was $6 \mathrm{~mm}$ in length) that covered the entire macular region. The scan that showed minimum foveal thickness was assumed to be the central SD-OCT scan, so acquisition was independent of patient fixation. Central foveal thickness was obtained by measuring the distance from the surface of the fovea to the high-reflectance layer, which corresponded to the retinal pigment epithelium-choriocapillaris complex (RPE/CC), and converting it into an actual value by using software of 3DOCT. In addition, cross-section scans were obtained through the point that was assumed to be the fovea. The IS/OS line was identified as a distinct band just above the RPE/CC line, and the line corresponding to the external limiting membrane (ELM) was detected just above the IS/OS line. We evaluated the status of both the IS/OS line and the ELM line in each eye, on both the horizontal and vertical scans through the centre of the fovea; only those cases in which the IS/OS line or the ELM line was definitely recognized on both the scans were defined as IS/OS $(+)$ or $\operatorname{ELM}(+)$. Those cases in which the IS/OS line or the ELM line was not partially recognized on either the horizontal or vertical scan were defined as IS/OS(-) or $\operatorname{ELM}(-)$. These measurements and evaluation were performed by a trained individual who was unaware of BCVA and of other pertinent information. We further divided the patients into two groups; one was with intact IS/OS lines in both eyes (IS/OS $(+/+)$ ) and the other was with an absent or an indefinite IS/OS line at least in either eye (IS/OS(-/)).

All values are presented as median and range and/or mean \pm SD. Statistical analysis was performed using the Fisher's exact test for categorical variables or the $t$-test for continuous variables for comparison of the two groups: IS/OS $(+)$ and IS/OS $(-)$ or IS/OS $(+/+)$ and IS/OS $(-/)$. All statistical analyses were performed with STATA software for Windows (version 9.02; StataCorp, Lakeway, TX, USA). $P$-values of $<0.05$ were considered to be statistically significant.

\section{Results}

We examined 24 eyes of 14 patients ( 5 women and 9 men) who ranged in age from 22 to 73 years (mean, $50 \pm 15$ years). The median and range of logMAR BCVA were 0.155 and from -0.176 to 1.699 (mean $0.31 \pm 0.51$ ), and those of foveal thickness were $156 \mu \mathrm{m}$ and from 52 to $184 \mu \mathrm{m}$ (mean, $148.2 \pm 32.0 \mu \mathrm{m}$ ). A total of 4 patients (7 eyes; $28.6 \%$ ) showed the complete type of Behçet's disease with all four major features (oral aphtha, genital ulceration, dermatitis, and uveitis), whereas 10 patients (17 eyes; $71.4 \%$ ) showed the incomplete type of Behçet's with three major features (oral aphtha, uveitis, and either genital ulceration or dermatitis). Duration of the symptoms of the ocular disease ranged from 4 to 43 years (mean, $11.7 \pm 10.8$ years). Three patients $(21.4 \%)$ were currently treated with cyclosporine, eight patients (57.1\%) with prednisolone, and seven patients (50\%) with colchicine. Only one patient was on no treatment at all. In some patients, retinal or choroidal atrophy, sheathing of retinal vessels, or optic disc atrophy were observed by funduscopy. The four highly reflective lines in the fovea corresponding to the ELM, IS/OS, an unidentified structure, and the RPE-Bruch's membrane complex were discernible clearly in SD-OCT images. Eighteen eyes (75\%) had an absent or an indefinite ELM line, six (25\%) had an intact ELM line at the central fovea. Nine eyes $(37.5 \%)$ had an absent or an indefinite IS/OS line (IS/OS(-) eye group), whereas fifteen $(62.5 \%)$ had an intact IS/OS line at the centre of the fovea (IS/OS $(+)$ eye group) (Figure 1). Figure 2 shows a representative patient (a 32-year-old man) who was diagnosed as having Behçet's disease 5 years ago. BCVA was 20/40 and foveal thickness was $116 \mu \mathrm{m}$. ELM and IS/OS lines were indefinable.

Tables 1 and 2 show differences in the clinical characteristics between the IS/OS(+) and IS/OS(-) eye 

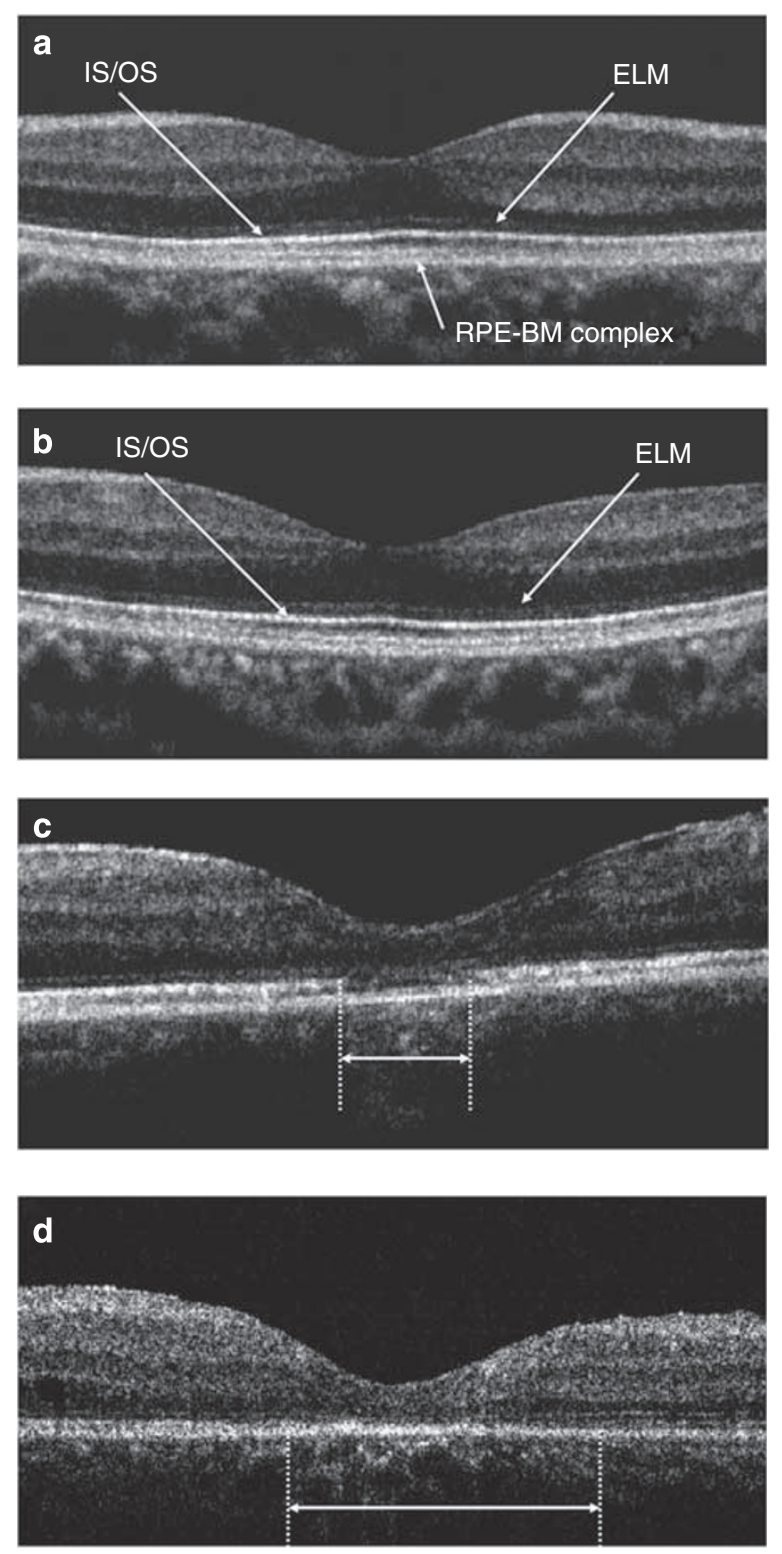

Figure 1 (a) A vertical SD-OCT image of a 50-year-old man normal subject. The four highly reflective lines in the fovea corresponding to the ELM, IS/OS, an unidentified structure, and the RPE-BM complex were identified. BCVA was 20/12.5. (b-d) SD-OCT sectional retinal images of fovea obtained with SD-OCT in the remission phase of Behçet's uveitis. (b) A horizontal SD-OCT image of a 35-year-old woman. BCVA was 20/12.5 and foveal thickness was $172 \mu \mathrm{m}$. In this scan, ELM and IS/OS lines were well identified. (c) A horizontal SD-OCT image of a 22-year-old man. This scan was defined as ELM(-) and IS/OS(-) because the ELM line and the IS/OS line at the fovea (doublesided arrow) were indefinite. BCVA was 20/50 and foveal thickness was $146 \mu \mathrm{m}$. (d) A vertical SD-OCT image of a 34-yearold man. A wide range of IS/OS line at the fovea (double-sided arrow) was invisible. BCVA was 20/50 and foveal thickness was $124 \mu \mathrm{m}$. RPE, retinal pigment epithelium; BM, Bruch's membrane. groups and IS/OS(+/+) and IS/OS(-/) patient groups. Male gender was more likely to have absence of the IS/OS line. LogMAR BCVA of the IS/OS(+) eye group was significantly better than of the IS/OS(-) eye group $(P=0.0001)$, and foveal thickness of the IS/OS $(+)$ eye group was significantly more than of the IS/OS(-) eye group $(P=0.0001)$. Figure 3 shows the box plots of $\operatorname{logMAR}$ BCVA for the IS/OS( + ) and the IS/OS(-) eye groups, and for the $\operatorname{ELM}(+)$ and the $\operatorname{ELM}(-)$ eye groups, with scatter-plot determined foveal thickness as the $x$ axis, and logMAR BCVA as the $y$ axis. BCVA of IS/OS( +) eye group was significantly better than that of IS/OS(-) eye group, whereas there was no significant difference in BCVA between $\operatorname{ELM}(+)$ and the $\operatorname{ELM}(-)$ eye groups. In addition, foveal thickness shows a strong negative correlation with logMAR BCVA.

\section{Discussion}

Behçet's uveitis is characterized by acute and recurrent ocular inflammation, involving especially the retinal vasculature. ${ }^{3}$ Repeated ocular attacks result in limited visual recovery and in irreversible alterations of the sensory retina - even after the inflammation is under control, which is not typical in the eyes with other types of uveitis. ${ }^{7}$ Recently, a number of articles have described the advantages of the newer OCT technologies. ${ }^{12}$ Acquisition speed and resolution have been improved dramatically by a novel technique known as Fourierdomain, or spectral-domain, detection. In the SD-OCT cross-sectional images, two lines that corresponded to the strong back-reflection from the ELM and IS/OS were observed, which is in agreement with findings of previous studies conducted on normal human and animal eyes. ${ }^{13,14}$ Using this technology, we investigate the correlation between VA and the status of each retinal layer during remission of Behçet's uveitis.

In the present study, foveal thickness in the IS/OS(-) eye group was significantly thinner than that in the IS/OS( + ) eye group. In active phase of Behçet's disease, drastic changes in macular such as macular oedema or retinal ischaemia could lead to the direct and persistent damage of the fovea. ${ }^{15-17}$ We assumed that lack of definition of the IS/OS line on the OCT images reflected disorganization of the photoreceptor layer, and that the decrease in foveal thickness reflected damage of either whole or partial retinal layers. From a different point of view, Atmaca and Sonmez ${ }^{6}$ reported the FA and ICGA findings in Behçet's disease. They suggested that the hyperfluorescence and/or hypofluorescence, irregular filling of the choriocapillaries, choroidal filling defect, and ICG leakage from choroidal vessels seen on ICGA represented choroidal involvement in Behçet's uveitis. Klaeger $e t a l^{5}$ also showed that the hypofluorescent 



Figure 2 Fundus photograph, fluorescein angiograms and SD-OCT images of a 32-year-old man. The diagnosis of Behçet's disease was made based on three major criteria (oral ulcers, genital ulcers, and ocular involvement) and his HLA typing was B51 at the age of 27 years. (a) Fundus photograph of the left eye in the remission phase shows the degenerative appearance of the fovea. Fluorescein angiogram in the active phase (b) shows leakage of fluorescein from the retinal vasculature, whereas the angiogram during remission (c) shows no fluorescein leakage. Horizontal (d) and vertical (e) SD-OCT scans through the fovea show discontinuous (double-sided arrow) or partially defective (arrowhead) IS/OS lines and obscure ELM lines. Best-corrected visual acuity was 20/40, and foveal thickness was $116 \mu \mathrm{m}$

Table 1 Individual eye characteristics by integrity of the IS/OS line

\begin{tabular}{|c|c|c|c|c|}
\hline & All & $I S / O S(+)$ & IS/OS(-) & P-value \\
\hline Number of eyes & 24 & 15 & 9 & \\
\hline Age (years) & $51.1 \pm 13.9$ & $55.4 \pm 12.2$ & $44.0 \pm 14.4$ & 0.052 \\
\hline Sex & & & & 0.033 \\
\hline Male & 14 & 6 & 8 & \\
\hline Female & 10 & 9 & 1 & \\
\hline BCVA (logMAR) & $0.31 \pm 0.51$ & $0.024 \pm 0.17$ & $0.77 \pm 0.54$ & 0.0001 \\
\hline Disease type & & & & 0.191 \\
\hline Complete (4 main symptoms) & 7 & 6 & 1 & \\
\hline Incomplete (3 main symptoms) & 17 & 9 & 8 & \\
\hline Duration of symptoms (years) & $12.1 \pm 11.2$ & $14.8 \pm 13.5$ & $8.1 \pm 5.4$ & 0.1722 \\
\hline \multicolumn{5}{|l|}{ OCT findings } \\
\hline Foveal thickness $(\mu \mathrm{m})$ & $148.2 \pm 32.0$ & $165.2 \pm 16.4$ & $119.9 \pm 32.1$ & 0.0001 \\
\hline Foveal ELM dropout & 18 & 9 & 9 & 0.052 \\
\hline Foveal IS/OS dropout & 9 & - & - & - \\
\hline \multicolumn{5}{|l|}{ Systemic medication } \\
\hline Cyclosporine & 6 & 2 & 4 & 0.150 \\
\hline Prednisolone & 15 & 9 & 6 & 1.000 \\
\hline Colchicine & 12 & 9 & 3 & 0.400 \\
\hline
\end{tabular}

The $P$-values $<0.05$ are statistically significant and are shown in bold. 
Table 2 Patient characteristics by integrity of the IS/OS line

\begin{tabular}{|c|c|c|c|c|}
\hline & All & $\operatorname{IS} / O S(+/+)$ & IS/OS(-/ ) & P-value \\
\hline Number of patients & 14 & 5 & 9 & - \\
\hline Age (years) & $50.2 \pm 14.9$ & $54.0 \pm 13.9$ & $48.1 \pm 15.7$ & 0.4997 \\
\hline $\operatorname{Sex}$ & & & & 0.023 \\
\hline Male & 9 & 1 & 8 & \\
\hline Female & 5 & 4 & 1 & \\
\hline BCVA (logMAR) in the worse eyes & $0.52 \pm 0.57$ & $0.040 \pm 0.21$ & $0.78 \pm 0.53$ & 0.0124 \\
\hline Disease type & & & & 0.095 \\
\hline Complete & 4 & 3 & 1 & \\
\hline Incomplete & 10 & 2 & 8 & \\
\hline Duration of symptoms (years) & $11.7 \pm 10.8$ & $16.8 \pm 17.6$ & $9.4 \pm 6.3$ & 0.2774 \\
\hline Foveal thickness $(\mu \mathrm{m})$ in the worse eyes & $138.9 \pm 35.1$ & $167.6 \pm 11.0$ & $123.0 \pm 33.9$ & 0.0155 \\
\hline \multicolumn{5}{|l|}{ Systemic medication } \\
\hline Cyclosporine & 3 & 0 & 3 & 0.258 \\
\hline Prednisolone & 8 & 3 & 5 & 1.000 \\
\hline Colchicine & 7 & 3 & 4 & 1.000 \\
\hline
\end{tabular}

Abbreviations: IS/OS $(+/+$ ), intact IS/OS lines in both eyes; IS/OS(-/ ), an absent or an indefinite IS/OS line in either eye.

The $P$-values $<0.05$ are statistically significant and are shown in bold.
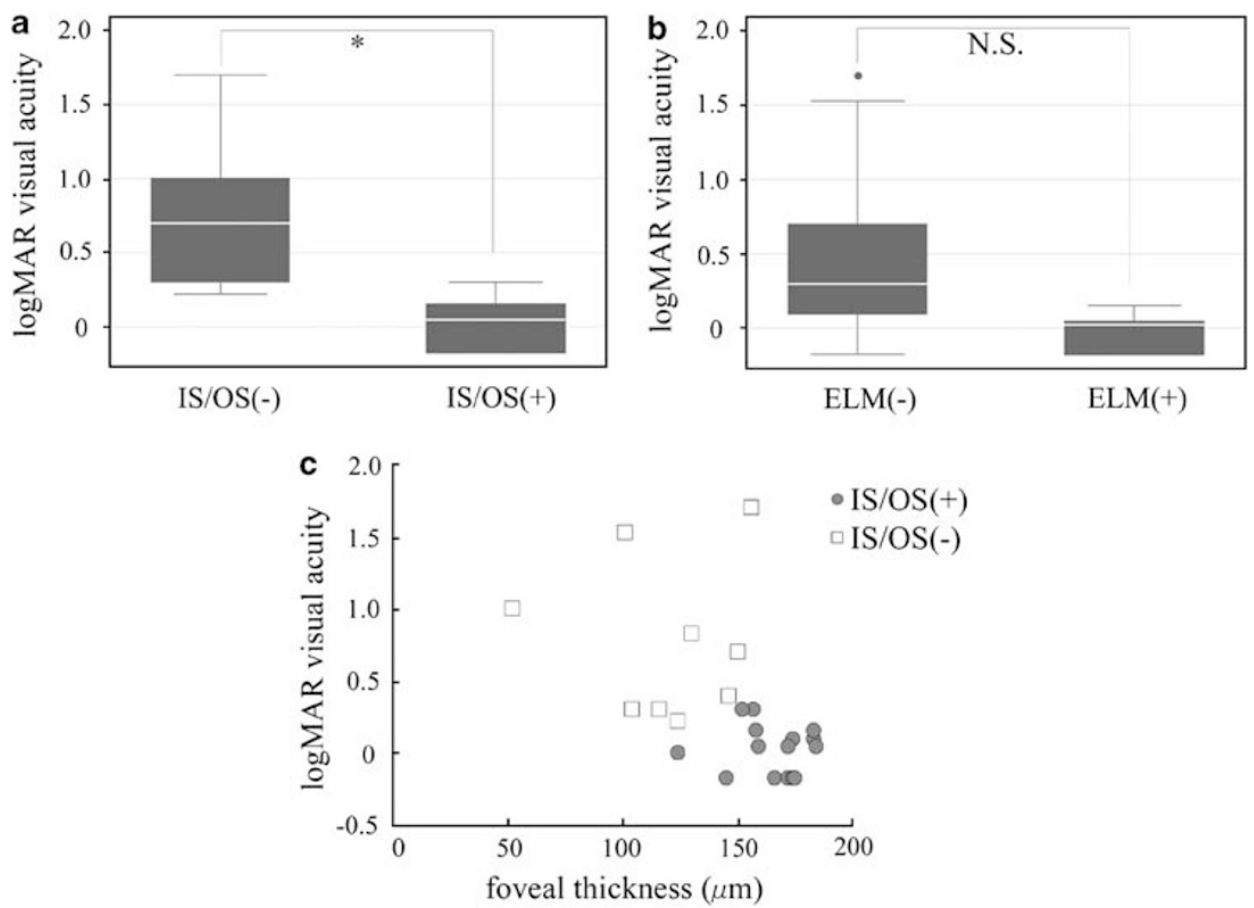

Figure 3 (a) The first box plot shows logMAR BCVA difference between the IS/OS(+) and IS/OS(-) eye groups. The difference was significant $(P=0.0001)$. (b) The second box plot shows logMAR BCVA difference between the $\operatorname{ELM}(+)$ and $\operatorname{ELM}(-)$; this difference was not significant $(P=0.071)$. (c) The scatter plot shows that the IS/OS( + ) eye group forms a separate colony from that of the IS/OS(-) eye group. The dots of the IS/OS(+) eye group are concentrated towards a lower logMAR BCVA and a thicker foveal thickness than that of the IS/OS(-) eye group.

lesions seen on all phase of ICGA, but not on FA, might represent chorioretinal stromal atrophy after choroidal inflammation. Because choroidal circulation has a major role in supplying the sensory retina with nourishment and oxygenation, damage to the choroidal vessels could cause disorganization of the photoreceptor layer in the retina. In addition, logMAR BCVA in the IS/OS(-) eye group was significantly worse than that in the IS/OS $(+)$ eye group. This supports the hypothesis that the IS/OS line detected by OCT reflects integrity of the photoreceptor layer, and, accordingly, vision of eyes with an ill-defined IS/OS line is poor, as has been shown 
previously in reports of several other retinal diseases about the relationship between visual acuity and the IS/OS line as seen by OCT, ${ }^{8-12}$ and we have now suggested that this may be the case in patients with Behçet's disease.

Second, the ratio of men to women in the IS/OSi $(+)$ eye group or the IS/OS $(+/+)$ patient group was significantly lower than that in the IS/OS(-) or the IS/OS(-/) patient group. Male gender and early age at onset of uveitis have been reported to be poor prognostic factors in Behçet's uveitis. ${ }^{18-21}$ The marked difference of the gender ratio might reflect a difference in severity of the disease between men and women.

Definition of the ELM, the boundary between photoreceptor bodies and inner segments in the fovea, was not correlated significantly with BCVA in the present study, although the BCVA of eyes with an intact ELM line tended to be better than that in eyes with an intermittent line. Eandi et $a l^{22}$ found an association between the inability to see the ELM in eyes with atrophic foveae and poor VA in patients with central serous chorioretinopathy. There is certainly a difference in target diseases, but repeated ocular attacks may cause alteration or obliteration of the structure of the ELM, the ability to visualize the ELM, or both. In our study, however, it is possible that the photoreceptor IS and OS were affected more than was the ELM by repeated ocular inflammation. It is also possible that the ELM itself (being harder to visualize on OCT than IS/OS layer) is more subject to error when grading of measurements is being carried out by masked observers.

Certainly, this study has several limitations including its small sample size and cross-sectional nature. We defined the scan that had shown minimum foveal thickness as the scan at the central fovea. However, it may be a source of error in patients with Behçet's disease, because prolonged inflammation could induce atrophic changes in retina and parafoveal retina could be thinner than central fovea as a result. Besides, ocular Behçet's disease is also characterized as optic nerve vasculitis, which would lead to ischaemic optic atrophy. Optic atrophy is also one of the considerable factors that influence visual acuity. Although we excluded the cases with optic disc paleness from the present study, it is possible that the cases with subclinical or retrobulbar optic nerve atrophy are included in the study subjects and they cause a kind of confounding factors. Moreover, the patients seen in this study were from a tertiary referral centre so that the patient population may consist of their own local catchment population as well as referrals from other centres that are likely to be biased towards more severely affected patients.

In conclusion, the IS/OS line, but not the ELM, detected by SD-OCT was correlated significantly with
BCVA in eyes with ocular Behçet's disease in remission. It is of great use in studying the histopathology of Behçet's uveitis, and of help in evaluating the status of the IS/OS line during remission for the prediction of visual outcome. Our findings suggest that the absence of an IS/OS line on OCT may be a useful marker for the irreversibility of visual loss, which would be a very useful sign for the clinician not to press ahead with further interventions that might carry risk of further harm. However, longitudinal studies of a large sample size are necessary to determine if a relationship exists between the foveal photoreceptor IS and OS layers and visual acuity in eyes with ocular Behçet's disease.

\section{Summary}

What was known before

- SD-OCT allows us to evaluate the junctions between inner and outer segments (IS/OS) of the photoreceptors, which have been reported to have a close relationship with visual function in various conditions.

What this study adds

- Details of the structural changes in Behçet's retina on SD-OCT have not been documented. We investigated the correlation between visual acuity and the status of each retinal layer during remission of Behçet's disease.

\section{Conflict of interest}

The authors declare no conflict of interest.

\section{Acknowledgements}

No funding was received for this study.

\section{References}

1 Nussenblatt RB. Uveitis in Behçet's disease. Int Rev Immunol 1997; 14: 67-79.

2 France R, Buchanan RN, Wilson MW, Sheldon Jr MB. Relapsing iritis with recurrent ulcers of the mouth and genitalia (Behçet's syndrome); review: with report of additional case. Medicine (Baltimore) 1951; 30: 335-355.

3 Chajek T, Fainaru M. Behçet's disease. Report of 41 cases and a review of the literature. Medicine (Baltimore) 1975; 54: 179-196.

4 Matsuo T, Sato Y, Shiraga F, Shiragami C, Tsuchida Y. Choroidal abnormalities in Behçet disease observed by simultaneous indocyanine green and fluorescein angiography with scanning laser ophthalmoscopy. Ophthalmology 1999; 106: 295-300.

5 Klaeger A, Tran VT, Hiroz CA, Morisod L, Herbort CP. Indocyanine green angiography in Behçet's uveitis. Retina 2000; 20: 309-314.

6 Atmaca LS, Sonmez PA. Fluorescein and indocyanine green angiography findings in Behçet's disease. $\mathrm{Br}$ J Ophthalmol 2003; 87: 1466-1468. 
7 Sakane T, Takeno M, Suzuki N, Inaba G. Behçet's disease. N Engl J Med 1999; 341: 1284-1291.

8 Kitaya N, Hikichi T, Kagokawa H, Takamiya A, Takahashi A, Yoshida A. Irregularity of photoreceptor layer after successful macular hole surgery prevents visual acuity improvement. Am J Ophthalmol 2004; 138: 308-310.

9 Villate N, Lee JE, Venkatraman A, Smiddy WE. Photoreceptor layer features in eyes with closed macular holes: optical coherence tomography findings and correlation with visual outcomes. Am J Ophthalmol 2005; 139: 280-289.

10 Murakami T, Tsujikawa A, Ohta M, Miyamoto K, Kita M, Watanabe $\mathrm{D}$ et al. Photoreceptor status after resolved macular edema in branch retinal vein occlusion treated with tissue plasminogen activator. Am J Ophthalmol 2007; 143: 171-173.

11 Ota M, Tsujikawa A, Murakami T, Kita M, Miyamoto K, Sakamoto A et al. Association between integrity of foveal photoreceptor layer and visual acuity in branch retinal vein occlusion. Br J Ophthalmol 2007; 91: 1644-1649.

12 Schocket LS, Witkin AJ, Fujimoto JG, Ko TH, Schuman JS, Rogers $\mathrm{AH}$ et al. Ultrahigh-resolution optical coherence tomography in patients with decreased visual acuity after retinal detachment repair. Ophthalmology 2006; 113: 666-672.

13 Costa RA, Calucci D, Skaf M, Cardillo JA, Castro JC, Melo Jr LA et al. Optical coherence tomography 3: automatic delineation of the outer neural retinal boundary and its influence on retinal thickness measurements. Invest Ophthalmol Vis Sci 2004; 45: 2399-2406.
14 Chen TC, Cense B, Miller JW, Rubin PA, Deschler DG, Gragoudas ES et al. Histologic correlation of in vivo optical coherence tomography images of the human retina. Am J Ophthalmol 2006; 141: 1165-1168.

15 Ozdal PC, Ortaç S, Taskintuna I, Firat E. Posterior segment involvement in ocular Behçet's disease. Eur J Ophthalmol 2002; 12: 424-431.

16 Gedik S, Akova Y, Yilmaz G, Bozbeyoglu S. Indocyanine green and fundus fluorescein angiographic findings in patients with active ocular Behcet's disease. Ocul Immunol Inflamm 2005; 13: 51-58.

17 Yilmaz G, Akova Y, Aydin P. Macular ischaemia in Behçet's disease. Eye 2000; 14: 717-720.

18 Tugal-Tutkun I, Onal S, Altan-Yaycioglu R, Huseyin Altunbas $\mathrm{H}$, Urgancioglu M. Uveitis in Behçet disease: an analysis of 880 patients. Am J Ophthalmol 2004; 138: 373-380.

19 Demiroglu H, Barista I, Dundar S. Risk factor assessment and prognosis of eye involvement in Behçet's disease in Turkey. Ophthalmology 1997; 104: 701-705.

20 Kitaichi N, Miyazaki A, Iwata D, Ohno S, Stanford MR, Chams H. Ocular features of Behçet's disease: an international collaborative study. Br J Ophthalmol 2007; 91: 1579-1582.

21 Power WJ, Pedroza-Seres M, Foster CS. Outcomes in anterior uveitis associated with the HLA-B27 haplotype. Ophthalmology 1998; 105: 1646-1651.

22 Eandi CM, Chung JE, Cardillo-Piccolino F, Spaide RF. Optical coherence tomography in unilateral resolved central serous chorioretinopathy. Retina 2005; 25: 417-421. 